\title{
Conhecimento das mulheres sobre métodos contraceptivos em um município do sul do Tocantins
}

\author{
Women knowledge on contraceptive methods in a southern Tocantins city \\ Conocimiento de las mujeres sobre los metodos anticonceptivos en una municipio en \\ Tocantins sur
}

Juliana Nascimento da Silva ${ }^{1 *}$, Edinalva Carvalho Rocha Rodrigues ${ }^{1}$, Denise Soares de Alcântara ${ }^{1}$, Laís Fernandes de Oliveira ${ }^{1}$, Cláudia Christina Ribeiro Guimarães Neri de Magalhães ${ }^{1}$, Julliana Dias Pinheiro ${ }^{1}$, Marília Pantoja Soares da Silva ${ }^{1}$, Mirelly da Silva Ribeiro ${ }^{1}$, Leandra Cristhyne de Souza Barros ${ }^{1}$, Nayara Pereira de Abreu'.

\begin{abstract}
RESUMO
Objetivo: Analisar o conhecimento sobre métodos contraceptivos de mulheres cadastradas no Programa de Planejamento Familiar de três Unidades Básicas de Saúde em um município da região Sul do Tocantins. Métodos: Realizou-se um estudo descritivo, exploratório com abordagem qualitativa. A amostra consistiu em 16 mulheres, entre 18 a 40 anos de idade. Os dados foram coletados por meio de entrevista semiestruturada, entre os meses de setembro e novembro de 2019. Os resultados foram analisados pela técnica de análise de conteúdo. Resultados: A maior parte das entrevistadas era casada, possuía filhos, conhecia e já havia utilizado algum tipo de método contraceptivo, relatou maior segurança no uso da pílula e preservativo masculino. Conclusão: $O$ estudo mostrou a necessidade constante de esclarecer a população feminina sobre métodos contraceptivos, assim como cada uma de suas particularidades, para que possam escolher e planejar o momento ideal para o nascimento de seus filhos.
\end{abstract}

Palavras-chave: Saúde da mulher, Conhecimento, Anticoncepção, Planejamento familiar.

\begin{abstract}
Objective: The study aimed to analyze the knowledge on contraceptive methods de women registered in the Family Planning Program of three Basic Health Units in a municipality in the southern area of Tocantins. Methods: A descriptive, exploratory study with a qualitative approach was done. The sample consisted of 16 women, between 18 and 40 years old. Data were collected through semi-structured interviews, from September to November 2019. The results were analyzed by the content analysis technique. Results: Most of the women interviewed were married, had children, they knew and had used some type of contraceptive method, reported greater safety in the use of the male pill and condom. Conclusion: The study showed the constant need to clarify the female population about contraceptive methods, as well as each of their particularities, so that they can choose and plan the ideal moment for the birth of their children.
\end{abstract}

Keywords: Women's health, Knowledge, Contraception, Family planning.

\section{RESUMEN}

Objetivo: Analizar el conocimiento sobre los métodos anticonceptivos de las mujeres registradas en el Programa de Planificación Familiar de tres Unidades Básicas de Salud en um municipio de la región sur de Tocantins. Métodos: Se llevó a cabo um estudio descriptivo y exploratorio con un enfoque cualitativo. La muestra estaba compuesta por 16 mujeres, entre 18 y 40 años de edad. Los datos se recopilaron a través

1 Universidade de Gurupi (UnirG). Gurupi - TO *E-mail: silvajuliana581@gmail.com 
de entrevistas semiestructuradas entre septiembre y noviembre de 2019. Los resultados fueron analizados por la técnica de análisis de contenido. Resultados: La mayoría de las mujeres entrevistadas estaba casada, tenía hijos, conocía y ya había utilizado algún tipo de método anticonceptivo, informó una mayor seguridad en el uso de la píldora y del preservativo masculino. Conclusión: El estudio demostró la necesidad constante de aclarar a la población femenina sobre los métodos anticonceptivos, así como sobre cada una de sus particularidades, para que puedan elegir y planificar el momento ideal para el nacimiento de sus hijos.

Palabras clave: Salud de la mujer, Conocimiento, Anticoncepción, Planificación familiar.

\section{INTRODUÇÃO}

O cuidado à Saúde da Mulher vem sendo edificado, no Brasil, com base em diversas políticas públicas de saúde. Até meados de 1970, a saúde da mulher era vista apenas em uma proporção procriativa, enfatizando-se apenas a atenção voltada ao ciclo gravídico-puerperal. A saúde pública defendia o natalismo e a medicina fortalecia a naturalização das desigualdades entre os sexos, reforçando o olhar da mulher como mãe (BRASIL, 2011).

Diante da recorrência dos altos índices de adoecimento das mulheres no Brasil, em 1984, foi elaborado pelo Ministério da Saúde (MS) o Programa de Assistência Integral à Saúde da Mulher (PAISM), com a finalidade de melhorar a atenção à saúde da mulher, que incluía ações educativas, preventivas, de diagnóstico, tratamento e recuperação, englobando a assistência à mulher em clínica ginecológica, no prénatal, parto e puerpério, no climatério, em planejamento familiar, Doenças Sexualmente Transmissíveis (DST), câncer de colo de útero e de mama, entre outros que fossem necessários para atender as individualidades das mulheres (BRASIL, 2011).

Segundo Domingues RM (2013), o PAISM consistiu em um importante divisor, inovando as políticas públicas, no que se refere a integralidade e equidade para com a saúde da mulher, visando um tratamento de maneira holística, abrangendo todas as fases do ciclo vital, e não somente o ciclo gravídico-puerperal, como era o enfoque antes da criação do programa.

A partir desse novo modelo, o planejamento familiar passou a garantir o livre acesso ao atendimento nos serviços de saúde, sendo dada plena liberdade de decisão ao casal quanto aos métodos de contracepção, pertencendo ao Estado o dever de apenas ofertar e garantir as orientações aos métodos contraceptivos e todo o acompanhamento necessário (SILVA CD, et al., 2013).

Hoje, encontra-se uma variedade de contraceptivos no mercado. Alguns demandam da mulher um entendimento sobre o seu corpo, como os métodos comportamentais, sendo eles a "tabelinha", o método de Ogino-Knous, o método de observação do muco cervical e o método de temperatura. Há também outros, como o coito interrompido, que requerem um maior controle por parte do homem. Porém, são métodos pouco incentivados devido à elevada chance de falha.

Os mais usados e encorajados pelo Planejamento familiar são os métodos de barreira, podendo ser o preservativo masculino e feminino, o diafragma e espermicidas. Dispõe-se ainda de métodos hormonais: anticoncepcionais orais, injetável mensal e trimestral, e pílulas de progestogênio.

Outro método é a contracepção de emergência, que previne gravidez indesejada após o ato sexual desprotegido. Contudo, este meio não exclui a probabilidade de um aborto, principalmente quando utilizado sem orientação e de forma repetitiva. E, para aqueles casais que não desejam ter mais filhos e que se enquadram dentro dos critérios estabelecidos pelo Ministério da saúde, são indicados métodos definitivos e irreversíveis, sendo eles a vasectomia para o homem e a laqueadura para a mulher (BARROS SMO, 2009).

Garantindo a oferta de anticoncepcionais, o direito de eleição do contraceptivo passou a ser primordial em relação a saúde e controle da fecundidade. Nesse contexto, o planejamento familiar tem como propósito geral garantir aos casais o direito básico à cidadania e à escolha de ter ou não filhos (BURATTI CB, et al., 2011). 
É importante enfatizar que o Programa de Planejamento familiar não se restringe somente ao fornecimento de medicamentos necessários à contracepção, há ainda, como fator indispensável, o esclarecimento sobre os eixos dessa política, para que as mulheres possam ser protagonistas da própria saúde, construindo seu bem-estar característico, o que acarretará melhores condições de vida em sociedade (ANDRADE EC e SILVA LR, 2009).

Além disso, ampliar o acesso de mulheres e homens às informações sobre as opções que existem dos métodos contraceptivos e suas vantagens e desvantagens é essencial para que suas escolhas sejam assertivas dentro de suas necessidades e realidades. Assim, essa ampliação possibilita a obtenção de conhecimento acerca dos métodos contraceptivos que são cientificamente comprovados e disponibilizados, tendo plena liberdade de escolha pelo que melhor atenda suas particularidades (OSIS MJD, et al., 2006). Para tanto, os profissionais de saúde juntamente com os serviços de saúde precisam assegurar que cheguem até as mulheres informações adequadas e acesso aos métodos de contracepção eficazes e seguros, permitindo a escolha do método mais apropriado (PENAFORTE MCL, et al., 2010)

Mediante essas informações, o objetivo deste estudo foi analisar o conhecimento sobre métodos contraceptivos das mulheres cadastradas no Programa de Planejamento Familiar de três Unidades Básicas de Saúde em um município da região Sul do Tocantins, visando contribuir com os profissionais de saúde para melhora na qualidade da assistência à saúde da mulher.

\section{MÉTODOS}

Trata-se de um estudo descritivo, exploratório com abordagem qualitativa, realizado em três Unidades Básicas de Saúde (UBS) em um município do Sul do Tocantins. Foram escolhidas três UBS, todas localizadas na área urbana. A coleta de dados ocorreu nos meses de setembro a novembro de 2019 nos dias de atendimento do Programa de Planejamento Familiar das UBS.

A amostra constitui-se de 16 mulheres de 18 a 40 anos, adscritas nas respectivas UBS, que estiveram em conformidade com os critérios de inclusão da pesquisa, dos quais eram: mulheres cadastradas no Planejamento Familiar, com idade entre 18 a 40 anos, e que aceitassem participar livremente da pesquisa, assinando o Termo de Consentimento Livre e Esclarecido. Foram excluídas da pesquisa mulheres histerectomizadas, que possuíam algum tipo de déficit cognitivo e aquelas que não aceitaram participar do estudo.

A coleta de dados foi executada pelas próprias pesquisadoras por meio de uma entrevista semiestruturada, gravada por um gravador. Foram feitos questionamentos sobre conhecimento dos métodos contraceptivos, dúvidas e receios sobre o uso desses métodos e o profissional envolvido na obtenção das respectivas informações. Cada entrevista durou cerca de 10 minutos. Para análise dos dados utilizou-se a técnica de análise de conteúdo (BARDIN L, 2011).

Foi realizada, primeiramente, a caracterização dos sujeitos da pesquisa, em seguida as falas obtidas foram transcritas na sequência, ordenadas, categorizadas e discutidas de acordo com o referencial teórico. A categorização resultou em três grupos: conhecimento sobre os métodos contraceptivos, dúvidas sobre o uso dos métodos contraceptivos e informações sobre os métodos contraceptivos.

O projeto de pesquisa foi aprovado pelo Comitê de Ética e Pesquisa sob o parecer $n^{\circ} 3.549 .422$. Todas as entrevistadas foram previamente esclarecidas acerca da pesquisa e em seguida foram assinados os Termos de Consentimento Livre e Esclarecido. Para garantir o sigilo da identidade das participantes estas foram identificadas por flores.

\section{RESULTADOS E DISCUSSÃO}

Das 16 mulheres entrevistadas, 25\% tinham idade entre 18 e 20 anos, 56,25\% tinham idade entre 20 e 30 anos, e 18,75\% entre 30 e 40 anos. Em relação ao estado civil, $43,75 \%$ eram casadas, $37,5 \%$ solteiras e $18,75 \%$ em união estável. Quanto ao número de filhos, $75 \%$ das mulheres tinham de 1 a 2 filhos, $12,5 \%$ de 3 a 4 filhos e 12,5\% não possuíam filhos. Quando questionadas se já haviam utilizado algum tipo de método 
contraceptivo em algum momento de suas vidas, quinze (15) mulheres afirmaram que sim, e apenas uma (1) relatou que não.

A partir da análise das falas, estas foram agrupadas em três categorias: conhecimento sobre os métodos contraceptivos, dúvidas sobre o uso dos métodos contraceptivos e informações sobre os métodos contraceptivos. A seguir, apresentaremos as considerações das entrevistadas.

\section{Categoria 1: conhecimento sobre os métodos contraceptivos}

Os métodos contraceptivos são recursos que podem ser comportamentais, medicamentosos ou cirúrgicos. Há métodos de uso específico feminino e masculino e dentre eles são classificados em métodos reversíveis e não reversíveis (BRANDÃO ER, 2017).

Nas respostas, a seguir, é possível observar que a maioria das mulheres questionadas conhece os principais tipos de contraceptivos como pílula, Dispositivo Intrauterino (DIU), preservativo, anticoncepcional injetável e até métodos definitivos.

“Conheço injetável, pílulas e preservativo" (Flor 1).

"Sei que tem o DIU, a injeção, os anticoncepcionais e o preservativo" (Flor 2).

"Anticoncepcional, DIU e camisinha" (Flor 3).

"Tem a camisinha, DIU, o remédio e a injeção" (Flor 4).

"Camisinha masculina, feminina, anticoncepcional em comprimido, vacina, DIU" (Flor 5).

"Sei do DIU, anticoncepcionais, laqueadura e preservativo" (Flor 6).

"Conheço a pílula, camisinha e o DIU" (Flor 7).

O preservativo masculino e feminino são os métodos mais conhecidos, embora o masculino tenha maior destaque (VASCONCELOS VN, 2019 e DIAS AR, 2018). Andrade EC e Silva LR (2009) expõem que o preservativo masculino e anticoncepcional oral foram os métodos mais citados pelas usuárias. Dados semelhantes são encontrados no presente estudo. Silva CD, et al. (2013) destacaram em sua pesquisa que, mesmo diante do avanço das informações, ainda há um desconhecimento acentuado em relação à variedade de contraceptivos existentes, sendo que os mais citados foram os anticoncepcionais orais e preservativo masculino.

Observa-se que, neste estudo, assim como nos dados apresentados por Penaforte MC, et al., (2010), o preservativo masculino está entre os métodos mais conhecidos e utilizados. Tal fato pode ser justificado devido ao seu baixo custo. Deve-se considerar que, além de método contraceptivo, o preservativo masculino e feminino é o meio de prevenção para infecções sexualmente transmissíveis (IST), o qual deve ter o uso amplamente incentivado (BRASIL, 2017). Nota-se ainda que, em nenhum momento, foi citado por alguma das participantes os demais métodos existentes sendo eles comportamentais, cirúrgicos ou mesmo contraceptivos de emergência, o que leva a inferir que elas não conhecem ou não recebem esclarecimentos sobre estes, logo há uma escolha limitada.

Sendo assim é imprescindível que a mulher seja melhor orientada quanto a todos os tipos de contraceptivos disponíveis no mercado, bem como indicações, contraindicações, vantagens, riscos e benefícios de cada um. Devem ser levados em conta alguns fatores, como a decisão da mulher, do casal ou mesmo do homem, bem como suas particularidades, aliados às condições individuais e circunstanciais dos utilizadores do método (SANTOS AA, et al., 2015). Medeiros TFR, et al. (2016) relatam em seu estudo que nenhuma das entrevistas mencionou a vasectomia como método contraceptivo, sendo este um procedimento cirúrgico reversível realizado em homens. Resultado semelhante pôde ser observado nessa pesquisa. Esses achados demonstram que ainda nos tempos de hoje há uma sobrecarga da mulher como a principal responsável pela contracepção. Fato este que pode ser mais bem esclarecido pelos profissionais de saúde no Programa de Planejamento Familiar. 


\section{Categoria 2: dúvidas sobre o uso dos métodos contraceptivos}

Foi verificado se as entrevistadas apresentavam dúvidas ou receios sobre o uso dos anticoncepcionais e obtiveram-se as seguintes respostas:

"Só de dois. O DIU por conta da infecção e os comprimidos que são feitos de farinha, porque estraga o útero. Não tenho nenhum receio não, só em relação a esses que falei, mas o uso sei como usar corretamente" (Flor 5).

"Sim e não, já usei anticoncepcional, mas ele me deu miomas e não me sinto bem com ele porque me fez mal. Já usei a camisinha também" (Flor 8).

"Sim. O único receio que tenho é que eu sei que se usar de má forma ele causa doenças né?" (Flor 1).

"Hoje não. Eu não uso por motivo de autoestima mesmo, prazer" (Flor 2).

"Mais ou menos. Tenho do anticoncepcional, tem muita conversa dos efeitos colaterais, de quando parar de tomar e querer engravidar e aí pode acontecer algum problema nesse intervalo" (Flor 3).

"Tem vez que sim, tem vez que não, tenho mais medo por causa da camisinha, os comprimidos tenho segurança com eles" (Flor 9).

“.... vejo que são eficazes se a mulher usar direitinho. Se for remédio, confio sim. Dúvidas até que não, tenho mais é receio mesmo" (Flor 6).

O contraceptivo precisa apresentar aspectos que garantam sua eficácia, aliada a habilidade de proteger contra gravidez indesejada e a segurança capaz de amparar ou não causar danos à saúde de quem o utiliza (POLI ME, 2016).

No estudo de Silva IC (2018), a maior parte das mulheres relatou não ter nenhuma dúvida quanto ao uso dos métodos contraceptivos e se sentem seguras quanto ao uso desses métodos. Como pode ser observado no presente estudo, as participantes apresentaram receio quanto a efetividade do método que faziam uso, devido aos efeitos colaterais, e informações inadequadas sobre as ações dos métodos. Observou-se ainda que apesar dessas participantes relatarem não ter nenhuma dúvida, os informes apresentados por elas não são coerentes. Estes achados remetem a uma certa fragilidade de conhecimento, o que leva, na maioria das vezes, a não utilização dos contraceptivos ou até mesmo o uso de maneira errônea.

Vale ressaltar que a mulher precisa se sentir bem com o método escolhido e que o anticoncepcional citado pela maioria das entrevistadas é, muitas vezes, usado sem nenhuma prescrição, orientação ou acompanhamento por profissional de saúde, obtidos com recursos próprios, o que facilita a presença de falha e consequentemente aumento de efeitos adversos (ANDRADE EC e SILVA LR, 2009). Achados semelhantes foram encontrados no estudo de Paz EC e Ditterich RG, (2009), no qual as mulheres entrevistadas sabiam da existência dos métodos contraceptivos, porém tinham informações bem confusas em relação às caraterísticas destes.

As dúvidas e receios podem ser minimizados se as usuárias forem mais orientadas quanto a todos os aspectos que envolvem os métodos contraceptivos. Além disso, é importante que as mulheres busquem se informar mais sobre o assunto e que os profissionais de saúde intensifiquem suas ações educativas no Programa de Planejamento Familiar. É preciso também desmistificar o real objetivo do Programa, que é não só assegurar a assistência à anticoncepção, mas promover ações educativas para que a mulher e/ou o casal possam decidir e planejar com segurança o momento ideal para ter ou não seus filhos (BRASIL, 2008).

Nota-se que há uma boa aceitação em relação ao uso dos contraceptivos, ainda que essas mulheres tenham algum tipo de dúvida ou receio, o que pode ser sanado intensificando as orientações, evitando uma gravidez não planejada e infecções sexualmente transmissíveis. 
Vale salientar que o ensino de boa qualidade tem como resultado mulheres bem esclarecidas, maior adesão e continuidade quanto ao uso desses métodos e, ainda, um aumento de sua eficácia.

\section{Categoria 3: informações sobre métodos contraceptivos}

O planejamento sexual e reprodutivo é condição importante para a saúde de homens e mulheres. Dessa forma, todos têm o direito à atenção e ao planejamento reprodutivo, ou seja, acesso aos métodos de anticoncepção e concepção, bem como às informações e ao acompanhamento por um profissional de saúde em um contexto de escolha livre e informada (RAMOS LA, 2018).

Quando questionadas se já tinham recebido orientações quanto aos métodos contraceptivos e sobre a fonte dessa informação, a maioria afirmou serem enfermeiros e médicos os principais informantes. Algumas ainda destacaram os agentes de saúde, a escola e estudantes de enfermagem.

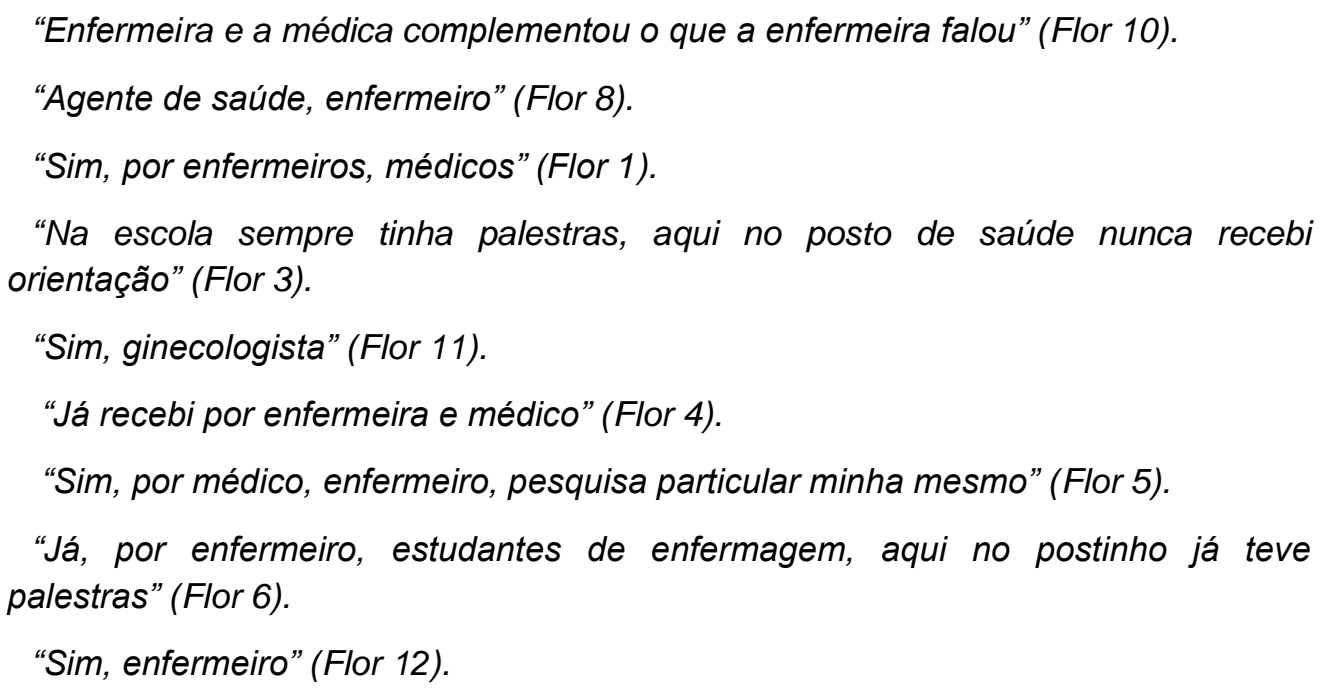

Silva IC (2018) apresenta em sua pesquisa que a principal fonte de informação para as mulheres sobre os métodos contraceptivos é o médico, em seguida os enfermeiros e, ainda, havia aquelas que relataram não ter recebido orientação alguma.

Andrade EC e Silva LR (2009) exibem o relato de mulheres que obtiveram informações através da amiga, vizinha, parente, conhecido e marido, e, em uma segunda proporção, citaram os médicos. Fator este bastante preocupante, pois é o profissional de saúde que pode melhor orientar em relação a este assunto.

No presente estudo, observa-se que o enfermeiro foi predominante no processo de transmissão do conhecimento. Isso mostra o quão importante é a atuação dessa classe, visto que, em geral, é o profissional que possui o convívio mais próximo e direto com o indivíduo, a família e a comunidade.

Sendo assim deve ser um profissional, sempre pronto a ouvir e acolher, aberto ao diálogo, de forma a estabelecer um vínculo de confiança para com a usuária, e, deste modo, fazer com que ela se sinta livre para escolher e interrogar se assim julgar necessário. O comportamento do profissional de saúde na assistência à anticoncepção deve englobar não só aconselhamento, mas, principalmente, ações educativas e práticas clínicas (PAZ EC e DITTERICH RG, 2009).

Para Poli ME (2016), é de suma importância que a equipe de saúde se prepare para atender e acolher a mulher, mediante as características clínicas de cada paciente, possibilitando o entendimento de todos os métodos contraceptivos, bem como suas características, efeitos, riscos, benefícios e eficácia.

Apesar dos serviços oferecerem cada vez mais equipes completas para atendimento nas Unidades Básicas de Saúde, esta pesquisa revelou que algumas entrevistadas não receberam nenhum tipo de orientação sobre os métodos contraceptivos, o que leva inferir que possam estar usando de maneira inapropriada, expondo-se a uma gravidez indesejada.

"Eu acho que não" (Flor 2). 


\author{
"Não" (Flor 7). \\ "Não" (Flor 9).
}

Destaca-se aqui a importância de explorar mais sobre este assunto de modo a esclarecer melhor as usuárias, permitindo que se sintam seguras quanto ao método escolhido e, ainda, o utilizem de maneira adequada, diminuindo, assim, as chances de falha e os danos à saúde.

Em geral, os profissionais devem estar continuamente empenhados junto aos propósitos do Planejamento Familiar para que haja modificações no comportamento das usuárias, resultando em excelentes marcadores de saúde, tal como melhora na qualidade de vida das mulheres.

O planejamento familiar é um território a ser trabalhado por esses profissionais que, se qualificados para desenvolver tais atividades propostas, podem assumir com autossuficiência o domínio deste Programa (ZUNTA RSB e BARRETO ES, 2014).

\title{
CONSIDERAÇÕES FINAIS
}

Com a realização deste estudo, constatou-se que as mulheres pesquisadas conhecem sobre os principais tipos de métodos contraceptivos disponibilizados na rede pública de saúde. A maioria já havia recebido algum tipo de esclarecimento sobre os métodos contraceptivos, embora ainda apresentassem dúvidas e receios sobre estes. Verificou-se que profissionais de saúde envolvidos no Programa de Planejamento Familiar realizam orientações sobre os métodos. Porém, percebeu-se a indispensabilidade de intensificar e ampliar as informações oferecidas sobre o assunto, tratando-o de forma clara e objetiva, a fim de que as mulheres possam se sentir seguras na escolha do método de contracepção e o utilizem de maneira adequada e consciente.

\section{REFERÊNCIAS}

1. ANDRADE EC, SILVA LR. Planejamento familiar: umaquestão de escolha. Rev.Eletr.Enf. v.11, n.1, p. 85-93, 2009.

2. BARDIN L. Análise de conteúdo. São Paulo: Edições 70, p. 229, 2011.

3. BARROS SMO. Enfermagemobstétrica e ginecológica: guia para a praticaassistencial. 2a ed. São Paulo: Roca; 2009.

4. BRANDÃO ER, et al. Os perigos submissos na contracepção de emergência: moralidade e saberes em jogo. Horiz.antropol., v.13, n.47, p. 131-161, 2017.

5. BRASIL. Ministério da Saúde. Secretaria de Políticas de Saúde. Área Técnica de Saúde da Mulher.

6. Assistência em Planejamento Familiar: Manual Técnico/Secretaria de Políticas de Saúde, Área Técnica de Saúde da Mulher. 4. ed. p 5-143 Brasília: Ministério da Saúde, 2008

7. BRASIL. Ministério da Saúde. Secretaria de Atenção à Saúde. Departamento de Ações Programáticas Estratégicas. Política Nacional de Atenção Integral à Saúde da Mulher: Princípios e Diretrizes. Brasília, 2011.

8. BRASIL. Ministério da Saúde. Departamento de Vigilância, Prevenção e Controle das IST, do HIV/Aids e das HepatitesVirais. 2017.

9. BURATTI CB, et al. Importância da Contracepção para o Planejamento Familiar e Prevenção da DST entre Graduandos de Enfermagem. Monografia (Enfermagem) - Universidade São Francisco, Bragança Paulista, 2011.

10. DIAS AR. As práticas de enfermagem na promoção da alto eficácia materna na amamentação no período pósparto. 2018. 116f. Mestrado (relatório de estágio). Escola superior de enfermagem do Porto de Saúde Materna e Obstetrícia. Porto, 2018.

11. DOMINGUES RM, et al. Acesso e utilização de serviços de pré-natal na rede SUS do município do Rio de Janeiro, Brasil. Rev. bras. epidemiol., São Paulo, v. 16, n. 4, p. 953-965, 2013.

12. MEDEIROS TRF, et al. Vivência de mulheres sobre contracepção na perspectiva de gênero. Revista Gaúcha de Enfermagem, v.37, n.2, jun, 2016.

13. OSIS MJD, et al. Atenção ao Planejamento Familiar no Brasil hoje: reflexões sobre os resultados de uma pesquisa. Cad. Saúde Pública, Rio de Janeiro, v. 22, n.11, p. 2481-2490, nov., 2006.

14. PAZ EC, DITTERICH RG. Conhecimento das mulheres sobre os contraceptivos no planejamento familiar. Rev. Gestão \& Saúde, Curitiba, v. 1, n. 1, p. 1-10. 2009.

15. PENAFORTE MCLF, et al. Conhecimento, uso eescolha dos métodos contraceptivos por um grupo de mulheres de uma Unidade Básica de saúde em Teresópolis, Rj. Cogitare Enfermagem, Rio de Janeiro, v.15, n.1, p. 124-130, janmar, 2010.

16. POLI ME. Anticoncepção. Manual de Ginecologia, 2016.

17. RAMOS LA, et al. Uso de Métodos Anticoncepcionais por Mulheres Adolescentes de Escola Pública. CogitareEnferm., v. 23, n. 3: e55230, 2018. 
18. SANTOS AA, et al. Fatores que interferem na escolha do método contraceptivo pelo casal: Revisão Integrativa. Rev APS, v. 18, n. 3, p. 368-377, jul-set, 2015.

19. SILVA CD, et al. Métodos Contraceptivos: conhecimento e prática de formandos em enfermagem. Rev. Enferm. UFPE online., Recife, v. 7, n. 11, p. 6322-8, nov., 2013.

20. SILVA IC, et al. Gravidez no puerpério: Conhecimento das mulheres quanto ao uso de métodos contraceptivos. Saber digital., v.11, n. 2, p. 35-41, 2018.

21. VASCONCELOS VN. Conhecimento das Mulheres sobre planejamento reprodutivo. 2019. 75f. Monografia (Enfermagem) - Universidade Federal do Maranhão - UFMA, São Luís, 2019.

22. ZUNTA RSB, BARRETO ES. Planejamento Familiar: critérios para escolha do método contraceptivo. Curso de Enfermagem da Universidade Paulista. São Paulo, v.32, n.2, p.173-178, 2014. 